\title{
Current Surgical Strategies for Lung Cancer with a Focus on Open Thoracotomy and Video-Assisted Thoracic Surgery
}

\author{
Kiyoshi Koizumi \\ Department of Surgery, Nippon Medical School
}

\begin{abstract}
The surgical treatment of the lung was developed primarily for pulmonary tuberculosis until the 1960s but was used for primary lung cancer in the 1970s. The incidence of lung cancer will increase in the next 30 years worldwide, and the annual incidence of lung cancer in Japan is expected to increase to about 150,000 by 2015 . Over the past 50 years, pulmonologists have performed clinicopathological studies in an attempt to prevent lung cancer. Early detection became possible with such studies; as a result, the rate of detection of lung cancer at stage I has increased. Furthermore, the frequencies of histological detection of peripheral squamous cell carcinoma and adenocarcinoma have increased. Thoracic surgeons have developed techniques, such as limited pulmonary resection, and have established a minimally invasive approach to the thorax. These successes were followed by the development of thoracoscopic surgery. However, minimally invasive surgery that allows for functional preservation has not yet been developed. Future investigations and the refinement of technologies are needed.
\end{abstract}

(J Nippon Med Sch 2006; 73: 116-121)

Key words: lung cancer, surgical treatment, thoracoscopic surgery, video-assisted thoracic surgery

\section{Introduction}

The history of surgery includes the investigation of limited surgery. Surgical treatment involves two opposing pursuits, namely, curability and functional preservation. Various trials and discussions regarding functional preservation and the pursuit of curability have continued since Graham reported on left pneumonectomy for primary lung cancer in $1933^{1-3}$. In 1960, Cahan ${ }^{4}$ recommended anatomic lobectomy with systemic mediastinal lymphadenectomy as "radical lobectomy" to achieve the balanced surgical effects of both curability and functional preservation. Subsequently, the verification of curability was evaluated according to mediastinal lymph node dissection and functional preservation with the development of limited surgery, and a minimally invasive approach to the thorax was investigated ${ }^{5-8}$. Since its introduction in the 1990s, thoracoscopic surgery has become widely used because of its minimal invasiveness ${ }^{9-12}$. However, whether thoracoscopic surgery truly has advantages for functional preservation and can

Correspondence to Kiyoshi Koizumi, MD, Division of Thoracic Surgery, Department of Surgery, Nippon Medical School, 1-1-5 Sendagi, Bunkyo-ku, Tokyo 113-8603, Japan

E-mail: sirakami@nms.ac.jp

Journal Website (http://www.nms.ac.jp/jnms/) 
achieve curability equal to that of conventional open thoracotomy remains unclear ${ }^{13-25}$.

\section{Change in Cause of Death: Pulmonary Tuberculosis and Lung Cancer}

The death rate from lung cancer surpassed that from pulmonary tuberculosis in the mid-1970s. Following the establishment of the Japanese Lung Cancer Society in 1960, a lung cancer screening research group was organized in 1972, and screening for lung cancer under the "Health and Medical Services Law for the Aged" was developed, established, and implemented nationwide. Lung cancer death rates for males and females were similar in 1998, and lung cancer became the most common cause of cancer death. Deaths from lung cancer have exceeded 50,000 and account for 18\% of all carcinoma deaths.

\section{Changes in Disease Stage and Histological Type of Primary Lung Cancer}

Advanced lung cancer accounted for $70 \%$ of disease of all stages in Japan in 1972 and 1986 but had markedly decreased by 1996, resulting in an increase in the percentage of patients with stage I or II disease following the nationwide implementation of a regular health examination system. In the last 5 years, more than $45 \%$ of cases of primary lung cancer have been detected through lung cancer screening examinations. However, the histological type of primary lung cancer has changed in the last 20 years $^{26}$. The incidence of adenocarcinoma increased markedly from 1982 1993 to 1993 2005. From 1993 to 2005, the nationwide incidences were $56 \%$ for adenocarcinoma, $33.0 \%$ for squamous cell carcinoma, $4 \%$ for large cell carcinoma, and $3 \%$ for small cell carcinoma. Central squamous cell carcinoma was previously the most common type of lung cancer but is now rare. Peripheral squamous cell carcinoma has become more common. This change has been attributed to the self-regulation of direct or indirect cancercausing factors such as smoking. In addition, I believe that the decrease in the incidence of central squamous cell carcinoma has a significant effect on the type of surgical procedure used.

\section{Change in Surgical Procedure}

Surgical procedures have changed according to changes in background features, such as disease stage, histological type, and the site of cancer progression. In 1999, 45\% of cases of primary lung cancer were detected by screening examinations, and the number of patients with small early lung cancer increased. On the other hand, with the increase in the average life span, an increase in the number of elderly patients with lung cancer has become a problem. Elderly patients often have concomitant diseases, such as ischemic heart disease and chronic pulmonary disease, which can be severe problems in the perioperative period. The period from 1970 to 1990 was characterized by a high frequency of extended resection for advanced lung cancer. On the other hand, over the past 10 years, the frequency of extended resection for advanced lung cancer has decreased and that of lobectomy with mediastinal lymphadenectomy by posterolateral thoracotomy (open thoracotomy) has markedly increased. Supported by these phenomena, minimally invasive surgery has become more popular over the last 10 years. Therefore, a technique that resects a smaller pulmonary volume and uses a minimally invasive approach to the thorax should be developed ${ }^{10-12}$.

Surgical treatment for lung cancer has been performed widely since Graham reported left pneumonectomy for primary lung cancer in $1933^{1}$. In 1951, Cahan reported that pneumonectomy and hilar lymphadenectomy are suitable for primary lung cancer, but that surgical stress could not be ignored ${ }^{2}$. In contrast, Churchill et al emphasized that lobectomy is less invasive than pneumonectomy but has a similar survival rate ${ }^{3}$. Finally, in 1960, Cahan recommended "radical lobectomy," consisting of lobectomy with systematic mediastinal lymphadenectomy, enabling curative treatment with functional preservation ${ }^{4}$. On the other hand, the preservation of physical condition was pursued by the development of limited surgeries, namely, 
segmental resection and wedge excision, and recovery after such surgeries ${ }^{6,8,13}$. The pursuit of functional preservation has continued with lobectomy through muscle-sparing thoracotomy and video-assisted thoracic surgery (VATS) with the accumulation of data from various cases ${ }^{9,14}$.

Over the past 25 years, my colleagues and I have performed surgery for elderly patients with lung cancer and have investigated clinical outcomes with respect to predicted postoperative pulmonary function. We have found differences in survival rate depending on differences in predicted postoperative pulmonary function, age, and physical reserve even in patients with stage I lung cancer. Therefore, we reviewed postoperative survival rates and the causes of death according to predicted postoperative pulmonary function. In patients older than 70 years survival rates differed significantly between patients with poor predicted postoperative pulmonary function and those with good predicted postoperative pulmonary function. However, in patients younger than 70 years, survival rates did not differ between these groups of patients. In contrast, when the same evaluation was performed in patients older than 70 years with stage I disease, only the results of patients with poor predicted pulmonary function were similar to those of patients with stage III lung cancer ${ }^{22}$. The mortality rate due to complications was $40 \%$ in patients with poor predicted postoperative pulmonary function. These findings clearly show the importance of pursuing treatment with preserved physical reserve by reducing invasiveness to the chest wall when performing surgery (not by open thoracotomy) for elderly patients with poor pulmonary function. When we reviewed chest wall structure again, we found that VATS causes less damage to the chest wall, including nerves, blood vessels, and bones, than does open thoracotomy. Injury to the chest wall, involving the nervous system, blood vessels, and bones, clearly differs depending on the approach to the thorax.

\section{Verification of Minimally Invasive Surgery}

Thoracoscopic surgery was introduced in Japan in 1992 and has been covered by the national health insurance since April 1, 1994. When we think about the future of surgical treatment, studies of the advantages and disadvantages of thoracoscopic surgery are important. In particular thoracic surgeons must consider what is achieved by thoracoscopic surgery for lung cancer. Investigations concerning the advantages and disadvantages of thoracoscopic surgery as a minimally invasive surgery are needed considering that lobectomy with mediastinal lymphadenectomy by open thoracotomy was not accepted as the standard surgery for lung cancer for 30 years. The verification of the advantages and disadvantages of thoracoscopic surgery for lung cancer starts with surgery-related, functional, and tumor-related factors.

1) Surgery-related factors: The duration of lobectomy with mediastinal lymphadenectomy by VATS is only slightly, but not significantly, longer than that with mediastinal lymphadenectomy by open thoracotomy; the durations of both surgeries become shorter with experience. However, blood loss during VATS is significantly less than that during lobectomy with mediastinal lymphadenectomy under open thoracotomy . However, intrapleural adhesion, chronic inflammation, and incomplete lobation influence the duration of surgery and blood loss. We compared the degree of chest wall damage by analyzing postoperative serum creatine phosphokinase (CPK/ $\mathrm{m}^{2}$ ) level in patients with or without these factors. We observed lower serum $\mathrm{CPK} / \mathrm{m}^{2}$ levels in patients who underwent VATS lobectomy with mediastinal lymphadenectomy than in patients who underwent lobectomy by open thoracotomy. This finding indicates that VATS is a minimally invasive procedure. The incidence and type of postoperative complication are similar between patients who underwent VATS and those who underwent open thoracotomy. However, complications were less severe in patients who underwent VATS. Univariate analysis showed that the duration of surgery is affected by the condition of the thoracic cavity. The Thoracoscopic Society ${ }^{9}$ recommends that thoracoscopic surgery should be stopped or be converted to open thoracotomy when surgery becomes lengthy. It is necessary for a thoracic 
surgeon to consider a switch to muscle-sparing thoracotomy as an option for minimally invasive surgery. Because VATS requires advanced technologies, it involves long and extensive training. In addition, the judgment that the advantage of VATS is lost at some point intraoperatively is important. According to analysis with receiveroperator characteristics curves for risk factors of VATS, surgery lasting more than 297 minutes offsets the advantages of $\mathrm{VATS}^{23}$. Even though VATS is a minimally invasive surgery, this finding shows that its advantages are lost if it is performed for a long time. When a thoracic surgeon observes adhesions caused by chronic inflammation in the thoracic cavity or calcified lymph nodes attached to a pulmonary artery, VATS must be converted to muscle-sparing thoracotomy. Another important factor to consider is that with a longer duration of surgery, blood loss increases.

2) Influence on changes in pulmonary function: To the maintain postoperative physical conditions, the preservation and prediction of postoperative pulmonary function are very important after surgical treatment. In particular, in lung cancer surgery, there are important factors that reduce functional lung volume. There are many reports on the loss or preservation of pulmonary function resulting from lung resection in patients who underwent open thoracotomy and those who underwent VATS. We investigated changes in pulmonary function before and 2,3 to 6,7 to 12 months after and 1 year or more after lung surgery in patients who underwent open lobectomy by open thoracotomy and those who underwent lobectomy by VATS. The rates of decrease in percent forced expiratory volume in 1 second (\% FEV1.0), percent forced vital capacity (\% VC), and percent maximal ventilatory volume (\% MVV) in patients who underwent VATS lobectomy were significantly low ${ }^{16}$. Furthermore, pulmonary function recovered more quickly after surgery in patients undergoing VATS lobectomy than in patients who underwent lobectomy by open thoracotomy. This faster recovery is also an important advantage in patients with severe pulmonary emphysema who underwent VATS $^{23}$ because lobectomy produces the same advantage as lung volume reduction surgery. This advantage is an additional benefit of VATS.

3) Effect on cardiopulmonary circulation : Pulmonary resection increases right ventricular afterload due to the loss of the pulmonary vascular bed resulting in chronic right ventricular insufficiency, which causes performance status and quality of life to deteriorate. Hemodynamics and right ventricular performance after pulmonary resection have been studied throughout the history of the surgical treatment of lung cancer ${ }^{27}$. Changes in hemodynamics and extravascular lung water have been reviewed with respect to age, disease stage, and difference in technique; however, the degree of right ventricular afterload differs depending on the patient's background even in patients who underwent the same surgical procedure. Therefore, thoracic surgeons must perform careful clinical bedside management. For the comparison of hemodynamics and right ventricular performance in the postoperative acute phase, VATS did not show an increase in systemic vascular resistance index greater than that with open thoracotomy. Patients who underwent VATS lobectomy showed a minimal increase in right ventricular afterload and an early recovery postoperatively, particularly elderly patients with a poor physical reserve ${ }^{24,25}$.

4) Postoperative inflammatory changes: A thoracic surgeon should know about postoperative inflammatory responses associated with extended stress owing to surgical intervention. Serum Creactive protein (CRP) levels are lower and changes in blood interleukin (IL)-6 levels are smaller in patients undergoing VATS lobectomy than in patients undergoing open thoracotomy. This finding suggests that postoperative inflammatory responses are weaker in VATS than in open thoracotomy. Furthermore, IL-8 levels in pleural effusion 6 hours after surgery in patients undergoing VATS lobectomy are one-seventh those in patients undergoing open thoracotomy ${ }^{17-19}$. Considering that IL-8 is a carcinoma growth factor as well as an inflammatory cytokine, VATS lobectomy seems to be advantageous with respect to invasiveness and tumor progression. 
5) Immunological responses: Immunosuppression associated with surgery may predispose patients to enhanced tumor growth or recurrence. Lymphocytes have a central immune network and respond to a particular marker by tumor immunosurveillance, which is a nonspecific reaction. Leaver and coworkers measured postoperative counts of lymphocytes, namely, CD4, CD8, and CD19, and natural killer $\mathrm{T}$ cells, with respect to immunosuppression, and found that invasive operations such as lobectomy for lung cancer have a strong effect on immunosuppression ${ }^{20,21}$. Our previous study showed that the degree of immunosuppression is smaller in VATS lobectomy than in open thoracotomy.

6) Changes in performance status before and after surgery: Deterioration of performance status after surgery is a subject of concern for patients, particularly the elderly. We have reported changes in performance status in elderly patients before and after surgery. Among patients undergoing VATS, only a small percentage show a postoperative decrease in performance status of more than 1 degree. The recovery speed and the maintenance of performance status were significantly better in patients undergoing VATS lobectomy than in patients undergoing open thoracotomy ${ }^{15,23-25}$.

7) Prognosis: One purpose of surgery for lung cancer is to improve the long-term prognosis. It is expected that VATS lobectomy cannot be accepted as a surgical treatment for lung cancer if it cannot achieve a survival rate equal to that of conventional open thoracotomy lobectomy. Whether equal mediastinal lymph node excision is feasible with VATS has been discussed for a long time among thoracic surgeons. The survival rate of lung cancer patients who undergo surgery is highest in Japan. An investigative committee formed by the Japan Lung Cancer Society and the Japanese Association for Chest Surgery found that the 5-year survival rates were $79 \%$ for stage IA, $60 \%$ for stage IB, $59 \%$ for stage IIA, $42 \%$ for stage IIB, $28 \%$ for stage IIIA, $20 \%$ for stage IIIB, and $19 \%$ for stage IV. The postoperative death rate was $3.0 \%: 1.4 \%$ postoperative mortality and $1.6 \%$ hospital death rates. The 5-year survival rates of patients who underwent surgical treatment at Nippon Medical School were $82 \%$ for stage IA, $67 \%$ for stage IB, $53 \%$ for stage IIA, 34\% for stage IIB, 39\% for stage IIIA, $11 \%$ for stage IIIB, and $23 \%$ for stage IV. Although the survival rates of patients who underwent VATS lobectomy are slightly higher according to data of other institutions, at Nippon Medical School, patients with pathological stage IA disease undergoing VATS lobectomy showed a 99\% 1-year survival rate, a 91\% 3-year survival rate, and a 91\% 5-year survival rate. There were no significant differences in these rates, i.e., the 93\% 1-year survival rate, the $86 \% 3$ year survival rate, and the $72 \% 5$-year survival rate, between patients who underwent lobectomy by open thoracotomy and those who underwent VATS. Even though VATS lobectomy cannot always be considered an excellent surgical treatment for lung surgery on the basis of its history, we believe that it is a good surgical treatment for lung cancer that should be developed further.

\section{Advantages and Disadvantages of VATS Lobectomy and Future Prospects}

We should avoid performing invasive surgical interventions that undermine the physical reserve of patients. We have shown the advantages and disadvantages of VATS lobectomy. The surgical treatment of lung cancer by VATS seems to be an ideal approach as minimally invasive surgery. Japanese thoracic surgeons who had 10 years' or more experience with conventional surgery switched to VATS lobectomy when it was introduced in Japan. There remains the issue of how to solve the major problems of this technique, such as safety and prognosis, in the future. At present, more than $20 \%$ of all pulmonary lobectomies are performed with VATS. Thoracic surgeons of the next generation must attain the skills of their seniors in a shorter time. Therefore, senior surgeons must provide an environment conducive for young surgeons to learn manual skills and acquire hands-on experience in operating new devices. We believe that more accurate VATS will be developed in the future.

Surgical strategies have changed according to 
patients' needs and improvements in surgical procedure and equipment have been made over the past 20 years. A custom-designed therapy based on research that is transrelational by basic, clinical medicine is promoted. However, surgeons should still focus on decreasing the invasiveness of surgical treatment for lung cancer.

\section{References}

1. Graham EA, Singer JJ: Successful removal of an entire lung for carcinoma. JAMA 1933; 101: 13711374.

2. Cahan WG: Radical pneumonectomy. J Thorac Surg 1951; 22: 449-473.

3. Churchill ED, Sweet RH, Scoutter L, et al.: The surgical management of carcinoma of the lung; A study of the cases treated at the Massachusets General Hospital from 1930-1950. J Thorac Surg 1950; 20: 349-365.

4. Cahan WG: Radical lobectomy. J Thorac Surg 1960; 39: $555-572$

5. Naruke T, Suemasu K, Ishikawa S: Surgical treatment for lung cancer with metastasis to mediastinal lymph nodes. J Thorac Cardiovasc Surg 1976; 71: 279-285.

6. Jensik RJ: Miniresection of small peripheral carcinoma of the lung. Surg Clin North Am 1987; 67: 951-958

7. Lung Cancer Study Group (Ginsberg RJ, Rubinstein LV): Randomized trial of lobectomy versus limited resection for T1N0 non-small cell lung cancer. Ann Thorac Surg 1995; 60: 615-623.

8. Mack MJ: Introduction and general techniques. Ann Thorac Surg 1993; 56: 609 .

9. Ginsberg RJ: Alternative (muscle sparing) incision in thoracic surgery. Ann Thorac Surg 1993; 56: 752754 .

10. Kohno T, Murakami T, Wakabayashi A: Anatomic lobectomy of the lung by means of thoracoscopy. An experimental study. J Thorac Cardiovasc Surg 1993; 105: 729-731.

11. Lewis RJ: The role of video-assisted thoracic surgery for carcinoma of the lung: Wedge resection to lobectomy by simultaneous individual stapling. Ann Thorac Surg 1993; 56: 762-768.

12. Wakabayashi A: Thoracoscopic partial resection in patients with severe chronic obstructive pulmonary disease. Arch Surg 1994; 129: 940-944.

13. Koizumi K, Akaishi T, Wakabayashi A: Anatomic segmental resection of the lung by thoracoscopy: An experimental study. Surgery Today 1997; 27: 10511055.

14. McKenna RJ Jr, Wolf RK, Brenner M, Fischel RJ, Wurnig P: Is lobectomy by video-assisted thoracic surgery an adequate cancer operation? Ann Thorac Surg 1998; 66: 1903-1908.

15. Koizumi K, Haraguchi S, Akiyama H, et al.: VATS lobectomy for primary lung cancer-Experiences based on provisional indications-. Surgery Today 1998; 28: 36-40.

16. Kaseda S, Aoki T, Hangai N, et al.: Better pulmonary function and prognosis with video-assisted thoracic surgery than with thoracotomy. Ann Thorac Surg 2000; 70: 1644-1646.

17. Yim AP, Wan S, Lee TW, Arifi AA: VATS lobectomy reduces cytokine responses compared with conventional surgery. Ann Thorac Surg 2000; 70: 243-247.

18. Sugi K, Kaneda Y, Esato K: VATS lobectomy reduces cytokine production more than conventional open thoracotomy. Jpn J Thorac Cardiovasc Surg 2000; 48: 161-165.

19. Nagahiro I, Andou A, Shimizu N: Pulmonary function after lobectomy, postoperative pain, and serum cytokine level after lobectomy: A comparison of VATS and conventional procedure. Ann Thorac Surg 2001; 72: 362-365.

20. Leaver HA, Craig SR, Yap PL, Walker WS: Lymphocyte responses following open and minimally invasive thoracic surgery. Eur J Clin Invest 2000; 30: 230-238.

21. Craig SR, Leaver HA, Yap PL, Pugh GC, Walker WS: Acute phase responses following minimal access and conventional thoracic surgery. Eur J Cardiothorac Surg 2001; 20: 455-463.

22. Koizumi K, Tanaka S, Haraguchi S, et al.: Evaluation of the prognosis of patients with stage I non-small cell lung cancer with respect to predicted postoperative lung function. Jpn J Thorac Surg 1996; 52: 162-168.

23. Haraguchi S, Koizumi K, Hatori N, et al: Postoperative respiratory complications of videoassisted thoracic surgery for lung cancer. J Nippon Med Sch 2004; 71: 30.

24. Mikami I, Koizumi K, Tanaka S: Changes in right ventricular performance in elderly patients who underwent lobectomy using video-assisted thoracic surgery for primary lung cancer. Jap J Thorac Cardiovasc Surg 2001; 49: 153-159.

25. Yamagishi S, Koizumi K, Shimizu K: Assessment of perioperative hemodynamics and right ventricular performance of lung cancer patient using continuous cardiac monitoring system; comparison between video-assisted thoracic surgery and muscle-sparing thoracotomy. Ann Thorac Cardiovasc Surg 2006; in press.

26. Nakada T: A study on the safety limit for the resection of lung cancer from the viewpoint of the cardiopulmonary reserve capacity that is based upon unilateral pulmonary artery occlusion with exercise. Lung \& Heart 1969; 3: 57-63.

27. Morita T, Sugano H: A statistical analysis of lung cancer registered in the Annual of Pathological Autopsy Cases in Japan between 1958 and 1987, with special reference to the characteristics of lung cancer in Japan. Acta Pathol Jpn 1990; 40: 665-675.

(Received, December 27, 2005)

(Accepted, April 25, 2006) 